Monográfico «Evaluación de la calidad en la educación superior»

PRESENTACIÓN

\title{
Las tecnologías de la información y la comunicación y la evaluación de la calidad en la educación superior
}

\author{
Gualberto Buela-Casal (coord.) \\ Angel Castro (coord.)
}

Fecha de presentación: marzo de 2009

Fecha de aceptación: abril de 2009

Fecha de publicación: julio de 2009

\begin{abstract}
Resumen
En este artículo introductorio al monográfico sobre «Evaluación de la calidad en la educación superior» se pone de manifiesto la importancia de las tecnologías de la información y la comunicación (TIC) en el contexto educativo universitario actual. En primer lugar se subraya la relevancia de la tecnología y la electrónica en el nuevo contexto globalizado y competitivo de hoy en día y se destaca el uso que las universidades hacen de las nuevas herramientas de comunicación, sobre todo de Internet. Posteriormente, se describe la importancia que muchos de estos instrumentos están adquiriendo en la evaluación de la calidad de la educación superior, a través de los cuales todos los días se llevan a cabo más procesos, análisis y auditorías. Por último, se relacionan ambas tendencias y se presenta el resto de artículos que forman parte del monográfico.
\end{abstract}

Palabras clave

tecnologías de la información y la comunicación, evaluación de la calidad, educación superior, universidades, globalización

\section{Information and communication technologies and quality evaluation in higher education}

\begin{abstract}
This introductory article for the monograph on "Quality Evaluation in Higher Education" demonstrates the importance of information and communication technologies (ICT) in the context of current university education. Firstly it underlines the relevance of technology and electronics in today's global and competitive context and highlights the use of the new tools for communication, particularly the Internet, by universities. Secondly it describes the importance that many of these tools are acquiring in quality evaluation in higher education, being used every day for more procedures, analyses and audits. Finally, it relates these trends and presents the rest of the articles in this monograph.
\end{abstract}

\section{Keywords}

information and communication technologies, quality evaluation, higher education, universities, globalization 
La globalización y los cambios que supone no son sólo económicos, sino también científicos, sociales y culturales. Se modifican las estructuras y la configuración de las sociedades como consecuencia de los movimientos migratorios entre todos los países del mundo. Este nuevo contexto globalizado se caracteriza también por la interacción entre países y sociedades a través de redes de comunicación más desarrolladas y eficientes (González Mariño, 2008), así como por la creación de instituciones, organismos e iniciativas transnacionales que conllevan cambios y adaptaciones en diversos ámbitos, como puede ser la creación del Espacio Europeo de Educación Superior (EEES). Las tecnologías de la información y la comunicación (TIC) han desempeñado y desempeñan un papel fundamental en todas las transformaciones que sufren las sociedades actualmente, generando beneficios en muchos sectores de ellas.

Como afirma Duart en las editoriales que la Revista de Universidad y Sociedad del Conocimiento (RUSC) publica en cada uno de sus números, en plena sociedad de la información la tecnología está cada vez más presente en todos los órdenes de la vida. Teléfonos, móviles, ordenadores y, sobre todo, Internet son herramientas básicas ya en la vida y, como tal, lo son para cualquier proceso de aprendizaje. Estas herramientas facilitan la búsqueda de información, el compartir esa información, generar conocimiento y compartirlo con personas a las que no conocemos, que se encuentran en otras partes del mundo. El conocimiento está cada vez más accesible, al alcance de cualquier persona y su gestión es el mayor desafío con el que se encuentran tanto las universidades como las propias TIC (Fainholc, 2006; Lara, 2009).

De estos instrumentos, sobre todo de Internet, se están sirviendo las universidades para mostrarse al mundo y ofrecer lo que cada una considera más relevante. En otra de sus editoriales, Duart informa de que todas las universidades españolas cuentan actualmente con presencia en la Red, y más del 60\% de ellas presta servicios directos a su comunidad universitaria. El reto actual, por tanto, no consiste en estar o no, sino en para qué estar, en qué ofrecer al mundo, en una ventana como el único contacto que personas de otros países pueden tener con tu universidad. La calidad es clave a la hora de tomar estas decisiones de política estratégica, por lo que se ha de cuidar lo que se transmite a través de las TIC (Roca, 2006). La evaluación de la calidad, por tanto, es esencial en este proceso, pero no sólo para la visibilidad de las universidades en el mundo (Aguillo y Granadino, 2006), sino también para mejorar la situación de las universidades en el contexto competitivo surgido tras la Declaración de Bolonia. Cuanto mejor sea la oferta de las universidades, mejor será la publicidad que se pueda hacer de ésta a través de Internet o de otros medios.

Son muchas las TIC y sus aplicaciones utilizadas en el ámbito educativo para mejorar la formación de los alumnos, profesores e investigadores. Las más utilizadas son, por supuesto, Internet, pero también los campus virtuales, la gestión informatizada de los expedientes o contenidos educativos, las plataformas electrónicas, los portales infomáticos o el e-learning a través de blogs y wikis (Dans, 2009; Franklin, 2006; Steegman, Huertas, Juan y Prat, 2008). También en los últimos años han aparecido muchas revistas electrónicas, como RUSC, que han tenido una gran aceptación y un buen funcionamiento, como afirman Abadal y Rius (2006). Son varias las críticas y recelos que despiertan las TIC, sobre todo en cuanto a su utilidad real para el alumnado, pues Ben Youssef y Dahmani (2008) estiman que es necesaria una adaptación de las estructuras universitarias para una mayor eficiencia de las TIC en el aprendizaje. También muestra preocupación por la cada vez mayor brecha digital que se observa entre aquellos que tienen acceso a las TIC y los que no (Ben Youssef y Ragni, 2008; Tello Leal, 2007), y pone empeño en no dejar de lado lo local, lo propio de cada universidad y su contexto dentro de este ámbito globalizado (de Oliveira, 2007). Lo cierto es que se ha de luchar por una mayor y mejor integración de las TIC en la metodología docente del alumnado -no sólo entenderlas como entretenimiento-, adaptándolas constantemente a una sociedad cambiante y teniendo siempre muy claro el gran poder de comunicación que se obtiene con ellas (Soto Carballo, 2007).

En cuanto a la calidad en la educación superior también se han observado cambios en su evaluación y en los principios inspiradores, sobre todo a consecuencia de la Declaración de Bolonia (1999) y del establecimiento de los objetivos y requisitos que los países miembros han de cumplir para la creación del Espacio Europeo de Educación Superior (EEES). Tradicionalmente, la evaluación de la calidad se realizaba teniendo en cuenta la productividad científica de las instituciones de educación superior, bien a través de las publicaciones de los profesores e investigadores (Buela-Casal, 2003, 2005; Buela-Casal et al., 2004), o bien a través del número de tesis doctorales defendidas en ellas (Agudelo et al., 2003; Moyano, Delgado y Buela-Casal, 2006). Pero en la actualidad y sobre todo tras la entrada de España en el EEES, la calidad en la educación superior es un concepto global, que implica a alumnado, profesorado, investigadores, departamentos, instituciones y al resto de personal involucrado en la vida universitaria (Buela-Casal y Castro, 2008; Del Río Bermúdez, 2008; Muñiz y Fonseca-Pedrero, 2008). Las 
universidades han de llevar a cabo programas de mejora globales para poder competir con otras instituciones de su entorno en este nuevo contexto competitivo, y ahí desempeñan un papel básico las TIC.

Con los cambios sufridos por el sistema universitario español en los últimos años, entre los que cabe resaltar la nueva legislación de los estudios de doctorado entendidos ahora como másteres oficiales, la creación de la mención de calidad para distinguir a los programas de doctorado que cumplen una serie de requisitos o la conversión de los títulos universitarios en grados - proceso que se lleva a cabo actualmente-, las tecnologías de la información y la comunicación han adquirido una importancia suprema. Actualmente, el correo electrónico es una herramienta fundamental para profesores e investigadores, que facilita el trabajo de personas en constante movilidad y que necesitan mantener el contacto con los miembros de sus grupos de investigación, así como facilita el intercambio con investigadores de todas las partes del mundo. Son tiempos en los que la Agencia Nacional de Evaluación de la Calidad y la Acreditación (ANECA) y las distintas agencias autonómicas cobran una gran relevancia en el desempeño de docentes e investigadores; en que se celebran con gran éxito cursos y congresos, como las distintas ediciones del Foro de Evaluación de la Calidad de la Educación Superior y de la Investigación, que cada año cuenta con más matriculados y con asistentes y ponentes más importantes y reconocidos; tiempos en que para escribir un artículo de investigación se ha buscar previamente en varias bases de datos internacionales. Además, hoy en día las solicitudes de becas, los proyectos de investigación, las acreditaciones y distintivos como la mención de calidad al doctorado se hacen casi íntegramente por vía electrónica mediante plataformas virtuales creadas por los distintos organismos -principalmente el Ministerio de Ciencia e Innovación- para dicho fin. Cada vez son más las publicaciones científicas que llevan a cabo todo el proceso de revisión electrónicamente, desde la recepción de los manuscritos hasta la publicación de los trabajos una vez revisados. De la misma manera, en muchas universidades el contacto que el docente tiene con sus alumnos conlleva un gran uso de herramientas similares, sobre todo plataformas electrónicas en las que se entregan y presentan trabajos, se consultan dudas y se ponen en común las preocupaciones de unos y otros.

Por todo esto, por la creciente y también necesaria relación entre la educación superior $-\mathrm{y}$ la evaluación de la calidad en ella- y las tecnologías de la información y la comunicación, se considera relevante el monográfico que aquí se presenta, formado por seis trabajos realizados por profesores e investigadores de gran relevancia poseedores del conocimiento y la autoridad para tratar los temas sobre los que escriben. En primer lugar, se presenta un trabajo titulado "Comparación de los indicadores de la calidad de las universidades», elaborado por miembros de la Agencia de Calidad, Acreditación y Prospectiva de las Universidades de Madrid (ACAP) y por el grupo de investigación dirigido por el Dr. Buela-Casal en la Universidad de Granada. En él se analizan y comparan los criterios utilizados por las agencias de evaluación de la calidad de los países con un mayor número de universidades consideradas las mejores del mundo según el ranking de Shangai. Posteriormente, se puede encontrar el artículo «Aplicación del análisis de valor para una evaluación integral y objetiva del profesorado universitario", realizado por un grupo de expertos en este tipo de análisis de la Universidad Politécnica de Cataluña y la Politécnica de Valencia. En tercer lugar, se encuentra un trabajo llevado a cabo por un grupo de investigación de la Universidad Complutense de Madrid, liderado por el Dr. Félix González Jiménez, titulado «Prospectiva y evaluación del ejercicio docente de los profesores universitarios como exponente de calidad».

Los tres últimos trabajos son igual de relevantes, ya que han sido elaborados por personas expertas en las materias que trabajan. Es el caso del artículo "Importancia de los criterios e indicadores de evaluación y acreditación del profesorado funcionario universitario en los distintos campos de conocimiento de la UNESCO», realizado por Juan Carlos Sierra, Gualberto Buela-Casal, M. ${ }^{a}$ Paz Bermúdez y Pablo Santos, de la Universidad de Granada y de «La evaluación de postgrados internacionales en la Unión Europea», llevado a cabo por Endika Bengoetxea y Jesús Arteaga, evaluadores de proyectos y del programa Erasmus Mundus en la Unión Europea. Por último, encontramos el trabajo titulado «Modelo de evaluación integral de la actividad docente, investigadora y gestora de los departamentos de la Universidad del País Vasco/ Euskal Herriko Unibersitatea», elaborado por Amaya Zárraga, Nekane Balluerka y Eva Ferreira, las cuales ocupan en la actualidad, o han ocupado, cargos de gobierno en la Universidad del País Vasco.

Para finalizar, se ha de agradecer a $R U S C$ la total disponibilidad mostrada para la publicación de este monográfico, el magnífico trato recibido por su equipo editorial y la relevancia de los contenidos que publica, en un ámbito importante, el de la relación entre la sociedad del conocimiento y la educación superior, en el que no hay muchas publicaciones en España. 


\section{Bibliografía}

AGUDELO, D.; BRETÓN-LÓPEZ, J.; ORTIZ-RECIO, G.; POVEDAVERA, J.; TEVA, I.; VALOR-SEgURA, I.; VICO, C. (2003). «Análisis de la productividad científica de la Psicología española a través de las tesis doctorales». Psicothema. Vol. I 5, pág. 595-6o9.

AGUILlO, I. F.; GRANADINO, B. (2006). «Indicadores web para medir la presencia de las universidades en la Red». Revista de Universidad y Sociedad del Conocimiento (RUSC). Vol. 3, n. ${ }^{\circ}$ I, pág. 68-75.

BEN YOUSSEF, A.; DAHMANI, M. (2008). "The impact of ICT on student performance in higher education: direct effects, indirect effects and organisational change». Revista de Universidad y Sociedad del Conocimiento (RUSC). Vol. 5, n. o I, pág. 45-56.

BEN YOUSSEF, A.; RAGNI, L. (2008). «Uses of information and communication Technologies in Europe's higher education institutions: from digital divides to digital trajectories». Revista de Universidad y Sociedad del Conocimiento (RUSC). Vol. 5, n. ${ }^{\circ}$ I, pág. 72-84.

BUELA-CASAL, G. (2003). «Evaluación de la calidad de los artículos y de las revistas científicas: propuesta del factor de impacto ponderado y de un índice de calidad». Psicothema. Vol. I 5, pág. 23-35.

BUELA-CASAL, G. (2005). "La evaluación de la calidad en el proceso de convergencia europea». Revista Mexicana de Psicología. Vol. 22, pág. 306-3 I 4.

Buela-CAsAl, G.; CAstro, A. (2008). «Análisis de la evolución de los programas de doctorado con mención de calidad en las universidades españolas y pautas para su mejora». Revista de Investigación en Educación. Vol. 5, pág. 49-6o

BUELA-CASAL, G.; MEDINA, A.; VIEDMA, M. I.; GODOY, v.; LOZANO, s.; TORRES, G. (2004). «Factor de impacto de tres revistas españolas de Psicología». Psicothema. Vol. I6, pág. 68 I-689.

DANs, E. (2009). «Educación online: plataformas educativas y el dilema de la apertura». Revista de Universidad y Sociedad del Conocimiento (RUSC). Vol. 6, n.o I, pág. 22-30.

DE OLIVEIRA, R. (2007). «Desterritorialización y localización de la enseñanza superior. En busca de un marco conceptual para la planificación de la enseñanza superior teniendo en cuenta el desarrollo regional». Revis- ta de Universidad y Sociedad del Conocimiento (RUSC). Vol. 4, n. ${ }^{\circ}$ 2, pág. 2-4.

«Declaración de Bolonia: El Espacio Europeo de la Enseñanza Superior». En: Reunión de Ministros Europeos responsables de Educación Superior. (I 9 de junio de i 999: Bolonia).

<http://web.micinn.es/contenido.asp?dir=04_Univers idades/o2 2 EdUnSu/o32EEES/or@Bolonia>

DEL Río BERMúdeZ, L. (2008). «Cómo implantar y certificar un sistema de gestión de la calidad en la Universidad». Revista de Investigación en Educación. Vol. 5 , pág. 5- I I .

FAINHOLC, B. (2006). «Rasgos de las universidades y de las organizaciones de educación superior para una sociedad del conocimiento, según la gestión del conocimiento". Revista de Universidad y Sociedad del Conocimiento (RUSC). Vol. 3, n. ${ }^{\circ}$ I, pág. I- - o.

FRANKLIN, T. (2006). «Portales en la educación superior: conceptos y modelos». Revista de Universidad y Sociedad del Conocimiento (RUSC). Vol. 3, n.o I, pág. 44-67.

GONZÁLEZ MARIÑo, J. C. (2008). «TIC y la transformación de la práctica educativa en el contexto de las sociedades del conocimiento». Revista de Universidad y Sociedad del Conocimiento (RUSC). Vol. 5, n. ${ }^{\circ}$, pág. I-8.

LARA, T. (2009). «El papel de la Universidad en la construcción de su identidad digital». Revista de Universidad y Sociedad del Conocimiento (RUSC). Vol. 6, n. ${ }^{\circ}$ I, pág. I 5-2 I.

MOYANO, M.; DELGADO, C. J.; BUEla-CASAL, G. (2006). «Análisis de la productividad científica de la Psiquiatría española a través de las tesis doctorales en la base de datos TESEO». International Journal of Psychology and Psychological Therapy. Vol. 6, pág. I I I-I 20.

MUÑIZ, J.; FONSECA-PEDRERO, E. (2008). "Construcción de instrumentos de medida para la evaluación universitaria». Revista de Investigación en Educación. Vol. 5, pág. I 3-25.

ROCA, G. (2006). «La presencia de las universidades en la Red». Revista de Universidad y Sociedad del Conocimiento (RUSC). Vol. 3, n. ${ }^{\circ}$ I, pág. I-5.

SOTO CARBALlo, J. (2007). «Políticas educativas y nuevos contextos de intervención en relación a las TIC. Panorama actual en el ámbito europeo y español». $R e-$ vista de Investigación en Educación. Vol. 4, pág. 4-2 I.

STEEGMAN, C.; HUERTAS, M.A.; JUAN, A. A.; PRAT, M. (2008). «E-learning de las asignaturas del ámbito matemáti- 
co-estadístico en las universidades españolas: oportunidades, retos, estado actual y tendencias». Revista de Universidad y Sociedad del Conocimiento (RUSC). Vol. 5 , n. ${ }^{\circ}$, pág. I-I 4 .
TELLO LEAL, E. (2007). «Las tecnologías de la información y comunicación (TIC) y la brecha digital: su impacto en la sociedad de México». Revista de Universidady Sociedad del Conocimiento (RUSC). Vol. 4, n.o 2, pág. I-8.

\section{Cita recomendada}

BUELA-CASAL, G.; CASTRO, A. (2009). «Las tecnologías de la información y la comunicación y la evaluación de la calidad en la educación superior». En: «Evaluación de la calidad en la educación superior» [monográfico en línea]. Revista de Universidad y Sociedad del Conocimiento (RUSC). Vol. 6, n. ${ }^{2}$. UOC. [Fecha de consulta: dd/mm/aa]. $<$ Dirección electrónica del PDF>

ISSN $1698-580 \mathrm{X}$

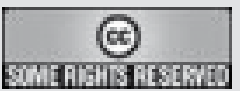

Los textos publicados en esta revista están sujetos -si no se indica lo contrario- a una licencia de Reconocimiento 3.0 España de Creative Commons. Puede copiarlos, distribuirlos, comunicarlos públicamente y hacer obras derivadas siempre que reconozca los créditos de las obras (autoría, nombre de la revista, institución editora) de la manera especificada por los autores o por la revista. La licencia completa se puede consultar en http://creativecommons.org/ licenses/by/3.0/es/deed.es. 


\title{
Sobre los autores
}

\author{
Gualberto Buela-Casal \\ Catedrático de Psicología \\ Universidad de Granada \\ Facultad de Psicología \\ Campus de Cartuja s $/ \mathrm{n}$ \\ I8orr Granada, España \\ gbuela@ugr.es
}

Licenciado en Psicología por la Universidad de Santiago de Compostela, doctor en Psicología por la Universidad Autónoma de Madrid, ha sido investigador del CIDE (Ministerio de Educación y Ciencia), profesor en las universidades de Málaga, Jaén, California y actualmente en la Universidad de Granada. Ha publicado más de 20 libros y más de i6o artículos en revistas científicas, en su mayoría en revistas indexadas en el Institute for Scientific Information (ISI). Ha dirigido 5 tesis doctorales. Es director de un programa de doctorado con mención de calidad: Diseños de investigación y aplicaciones en psicología y salud, distinguido recientemente con el premio AUIP a la calidad del doctorado. Actualmente es presidente de la Asociación Española de Psicología Cognitivo Conductual y director del Foro de Evaluación de la Calidad de la Educación Superior y de la Investigación.

\section{Angel Castro}

Licenciado en Ciencias Políticas y Sociología
Universidad de Granada
Facultad de Psicología
Campus de Cartuja s $/ \mathrm{n}$
I8orr Granada, España
castroa@ugr.es

Licenciado en Ciencias Políticas y Sociología (especialidad Sociología) por la Universidad de Granada, posee un máster oficial en Diseños de investigación y aplicaciones en psicología y salud por la misma universidad. Es coautor de varios artículos publicados en revistas del Journal Citation Reports y de otros publicados en revistas nacionales e internacionales. Además, ha participado en varias reuniones científicas nacionales e internacionales y ha realizado estancias de investigación en centros nacionales. Actualmente, es miembro del Departamento de Psicología Evolutiva y de la Educación de la Universidad de Granada y es profesor en el Campus de Melilla de dicha universidad, mientras desarrolla su tesis doctoral.

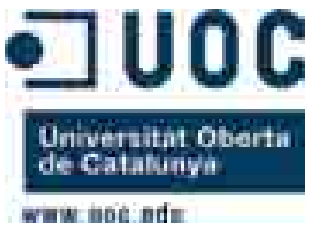

